\title{
Hyperon ordering in neutron star matter
}

\author{
L. Mornas*, J.P. Suárez Curieses* and J. Diaz Alonso*† \\ *Departamento de Fisica, Universidad de Oviedo, E-33007 Oviedo (Asturias) Spain \\ ${ }^{\dagger}$ LUTH, FRE2462 CNRS, Observatoire de Paris-Meudon, F92195 Meudon, France
}

\begin{abstract}
We explore the possible formation of ordered phases in neutron star matter. In the framework of a quantum hadrodynamics model where neutrons, protons and Lambda hyperons interact via the exchange of mesons, we compare the energy of the usually assumed uniform, liquid phase, to that of a configuration in which di-lambda pairs immersed in an uniform nucleon fluid are localized on the nodes of a regular lattice. The confining potential is calculated self-consistently as resulting from the combined action of the nucleon fluid and the other hyperons, under the condition of beta equilibrium. We are able to obtain stable ordered phases for some reasonable sets of values of the model parameters. This could have important consequences on the structure and cooling of neutron stars.
\end{abstract}

\section{INTRODUCTION}

The equation of state in the interior of neutron stars is usually considered to be that of an interacting Fermi liquid in a uniform phase. Under certain conditions however, a crystallized phase may be energetically more favorable. This in turn can have interesting consequences on the structure and evolution of the neutron stars. We may quote as examples thereof $(i)$ triaxial configurations with emission of gravitational waves [1], (ii) modification of the oscillation modes of the star [2] or (iii) of the neutrino transport properties [3].

In a previous paper [ 4 ] we presented the results pertaining to a simplified model where we considered three baryonic species: the neutron, proton and Lambda hyperon, interacting with each other through the exchange of $\sigma$ and $\omega$ mesons. We found that it was possible to find some sets of the model parameters such that the energy of a configuration where pairs of $\Lambda$ hyperons with antiparallel spins located at the nodes of a cubic lattice, could be energetically more favorable than the corresponding liquid phase.

The model presented in [ 7 w was a strictly minimal one. Here we would like to perform again these calculations with a more realistic description of the nuclear interaction. In order to reproduce the correct values of the incompressibility modulus and the asymmetry energy, we choose an approach based on the Density Dependent Hadron Field theory (DDRH) developped by the Giessen group [5, 6, 7]. In this model, the nucleon-meson vertices have a functional dependence on the density operator. In this way, some basic features of the short-range correlations are taken into account. 


\section{MODEL OF BARYONIC MATTER}

\section{Lagrangian}

We describe the nuclear interaction in the framework of a phenomenological quantum relativistic model. The interaction piece of the Lagrangian density in the Density Dependent Hadron Field Theory (DDRH) reads:

$$
\mathscr{L}_{\text {int }}=\sum_{B=N, H} \Gamma_{\sigma B} \bar{\psi}_{B} \sigma \psi_{B}-\Gamma_{\omega B} \bar{\psi}_{B} \gamma^{\mu} \omega_{\mu} \psi_{B}+\Gamma_{\delta B} \bar{\psi}_{B} \delta \psi_{B}-\Gamma_{\rho B} \bar{\psi}_{B} \gamma^{\mu} \rho_{\mu} \psi_{B}
$$

where the couplings $\Gamma_{\alpha B}$ are density-dependent through their functional dependence on the baryonic current $J^{\mu}$.

$$
\Gamma_{\alpha B}=\Gamma_{\alpha B}\left[\hat{J}^{\mu} \hat{J}_{\mu}\right] \quad \text { with } \quad \hat{J}^{\mu}=\sum_{B} \hat{\psi}_{B} \gamma^{\mu} \hat{\psi}_{B}
$$

We will consider only one species of hyperons, the $\Lambda$. In a realistic model, it can be expected that the $\Sigma$ hyperon will also play a role. However, the interaction of this hyperon with nuclear matter is less well known, so that it has not yet been implemented in the framework of a DDRH-type model.

\section{Liquid phase}

In the liquid phase we impose the usual conditions for charge neutrality and chemical equilibrium under $\beta$ decay among the baryons (neutron, proton, $\Lambda$ hyperon) and leptons (electron, muon) taken into account in our model.

$$
n_{e}+n_{\mu}=n_{p}, \quad \mu_{n}=\mu_{\Lambda}, \quad \mu_{p}-\mu_{n}=\hat{\mu}=\mu_{e}=\mu_{\mu}
$$

These equations determine the relative fraction of each species of particles. The equation of state is then obtained from the energy momentum tensor. For details we refer the reader to [5, 6, 7]. In particular we will be interested in comparing the energy density of the liquid phase $\rho_{L}=T^{00}$ to its counterpart in the crystallized phase.

\section{Self consistent confining potential}

We now assume that the hyperons are ordered on a lattice and are localized in gaussian clouds. We further assume that there are two hyperons with antiparallel spins per lattice site. This is partly in order to avoid complexities related to spin-spin interaction, but we can also justify this choice by arguing that the $\Lambda$ - $\Lambda$ interaction, as inferred from data on double hypernuclei, is attractive and may even favor a bound state ( $\sim \mathrm{H}$-dibaryon). In the spirit of the Sommerfeld aproximation, we neglect the redistribution of the surrounding nucleons. 
The potential energy of an hyperon around a lattice site is calculated as the sum of the interaction potential energies generated by hyperons at other lattice sites $\vec{r}_{i}=$ $a(l \vec{i}+m \vec{j}+n \vec{k})$.

$$
U_{\text {sup }}(\vec{r})=\sum_{i} U\left(\vec{r}-\vec{r}_{i}\right)
$$

In this expression, the potential energy of each hyperon is obtained by taking the convolution

$$
U(\vec{r})=\frac{2 \pi}{r} \int_{0}^{\infty} R n(R) d R \int_{|r-R|}^{|r+R|} V^{O B E}(x) x d x
$$

of a gaussian distribution

$$
n(r)=2 \Psi_{\Lambda}^{*}(r) \Psi_{\Lambda}(r)=2\left(\frac{M_{\Lambda} v_{0}}{\pi}\right)^{3 / 2} e^{-M_{\Lambda} v_{0} r^{2}}
$$

with the elementary one boson exchange potential $V^{O B E}(x)$ corrected for the finite size of hyperons by form factors (with cutoffs $\Lambda_{\sigma}, \Lambda_{\omega}$ ) and for relativistic effects in the momentum expansion. Note that in the present configuration, only the central and spinspin components contribute.

The potential calculated in this way is then approximated by a parabola $U_{\text {par }}$ parameterized by its depth $U_{0}$ and the frequency of oscillation of the hyperons in the potential well $v_{0}=\sqrt{\left.\nabla^{2} U_{\mathrm{par}}(\vec{r})\right|_{r^{\prime}=0} / M_{\Lambda}}$. The width $\Gamma=1 / \sqrt{M_{\Lambda} v_{0}}$ of the gaussian distribution (5) is determined self-consistently by the frequency of this harmonic oscillator.

\section{Equation of state of the ordered phase}

We again impose the conditions for $\beta$ equilibrium

$$
\mu_{n}=\mu_{p}+\mu_{e}, \quad \mu_{n}=\mu_{\Lambda}, \quad \mu_{e}=\mu_{\mu}
$$

with the chemical potentials given by

$$
\begin{aligned}
& \mu_{p}=\sqrt{p_{f p}^{2}+M_{p}^{2}}+\Gamma_{\omega N}\left(<\omega^{0}>+<\omega_{\Lambda}>_{s}\right)+\Gamma_{\rho N}<\rho^{0}>+\Sigma_{\text {rearr }}^{N} \\
& \mu_{n}=\sqrt{p_{f n}^{2}+M_{n}^{2}}+\Gamma_{\omega N}\left(<\omega^{0}>+<\omega_{\Lambda}>_{s}\right)-\Gamma_{\rho N}<\rho^{0}>+\Sigma_{\text {rearr }}^{N} \\
& \mu_{\Lambda}=M_{\Lambda}+U_{0}+\frac{5}{2} v_{0}+\Gamma_{\omega \Lambda}<\omega^{0}>+\Sigma_{\text {rearr }}^{\Lambda}
\end{aligned}
$$

The nucleons evolve in constant mean fields consisting of two contributions: To the field produced by the homogeneous nucleon fluid $\left(e . g . \omega^{0}\right)$, we add the spatial average of the fields generated by the periodic hyperon distribution $\left(e . g .<\omega_{\Lambda}>_{s}\right)$. The chemical potential of the hyperons is now determined by the parameters of the confining potential. The rearrangement terms $\Sigma_{\text {rearr }}$ contain derivatives of the couplings $\Gamma_{\alpha B}$ with respect to the density. 
We further impose the conditions of charge neutrality $n_{\mu}+n_{e}=n_{p}$, and express the baryonic density $n_{B}=n_{n}+n_{p}+n_{\Lambda}$ as the sum of the nucleon densities and the hyperon density, the latter being related to the lattice parameter $a$ by $n_{\Lambda}=2 / a^{3}$. Finally, we write the defining equations for the effective baryon masses and the self consistent equations obeyed by the fields $\sigma, \omega^{0}, \rho^{0}$ and $\delta$.

$$
\begin{aligned}
M_{p}= & m_{N}-\Gamma_{\sigma N}\left(<\sigma>+<\sigma_{\Lambda}>_{s}\right)-\Gamma_{\delta}<\delta> \\
M_{n}= & m_{N}-\Gamma_{\sigma N}\left(<\sigma>+<\sigma_{\Lambda}>_{s}\right)+\Gamma_{\delta}<\delta> \\
M_{\Lambda}= & m_{\Lambda}-\Gamma_{\sigma \Lambda}<\sigma> \\
& <\sigma>=\Gamma_{\sigma N}\left(n_{p}^{(s)}+n_{n}^{(s)}\right)=\frac{\Gamma_{\sigma N}}{m_{\sigma}^{2}} \sum_{i=p, n} \frac{M_{i}}{2 \pi^{2}}\left[p_{f i} \varepsilon_{f i}-M_{i}^{2} \ln \left(\frac{p_{f i}+\varepsilon_{f i}}{M_{i}}\right)\right] \\
& <\delta>=\Gamma_{\delta N}\left(n_{p}^{(s)}-n_{n}^{(s)}\right), \quad<\sigma_{\Lambda}>_{s}=\frac{\Gamma_{\sigma \Lambda}}{m_{\sigma}^{2}} n_{\Lambda} \\
& <\omega^{0}>=\frac{\Gamma_{\omega N}}{m_{\omega}^{2}}\left(n_{n}+n_{p}\right)=\frac{\Gamma_{\omega N}}{m_{\omega}^{2}}\left(\frac{p_{f p}^{3}}{3 \pi^{2}}+\frac{p_{f n}{ }^{3}}{3 \pi^{2}}\right) \\
& <\rho^{0}>=\frac{\Gamma_{\rho N}}{m_{\rho}^{2}}\left(n_{p}-n_{n}\right), \quad<\omega_{\Lambda}>_{s}=\frac{\Gamma_{\omega \Lambda}}{m_{\omega}^{2}} n_{\Lambda}
\end{aligned}
$$

The equations for chemical equilibrium (6),(7),(8) again determine the chemical composition of the matter at a given baryonic density. They have to be solved self consistently with the equations for the confining potential (3), to which they are interrelated through the effective hyperon mass, $M_{\Lambda}$ the lattice parameter $a$, the depth $U_{0}$ and the oscillation frequency $v_{0}$.

\section{NUMERICAL RESULTS}

We will use the rational parametrization of the coupling constants suggested in Ref. [8] with the parameters of Ref. [5]

$$
\Gamma_{\alpha N}\left(n_{B}\right)=a_{\alpha}\left[\frac{1+b_{\alpha}\left(\frac{n_{B}}{n_{\mathrm{sat}}}+d_{\alpha}\right)}{1+c_{\alpha}\left(\frac{n_{B}}{n_{\mathrm{sat}}}+e_{\alpha}\right)}\right], \quad \Gamma_{\alpha \Lambda}\left(n_{B}\right)=x_{\alpha \Lambda} \Gamma_{\alpha N}\left(n_{B}\right)
$$

This function is chosen so as reproduce parameter free Dirac-Brueckner calculations in the nucleon sector. The parameters $x_{\alpha B}$ are adjusted in order to reproduce the binding energy of the $\Lambda$ in hypernuclei. The coupling constants used in the present work correspond to the "model 1 with DD phenomenological" parametrization of Reference [7]. We would like to stress the fact that, in contrast to the calculation performed in [卂], we have essentially no free parameters in the results presented in this work.

When numerical convergence is reached, we obtain the parameters $U_{0}, v_{0}, M_{i}, p_{F i}$, etc. which fully determine the thermodynamical state of the system. We now are in a position to calculate the energy density of the crystallized phase. It is obtained as

$$
\rho_{C}=\rho_{p}+\rho_{n}+\rho_{e}+\rho_{\mu}+\rho_{\Lambda}+\rho_{\text {fields }}
$$




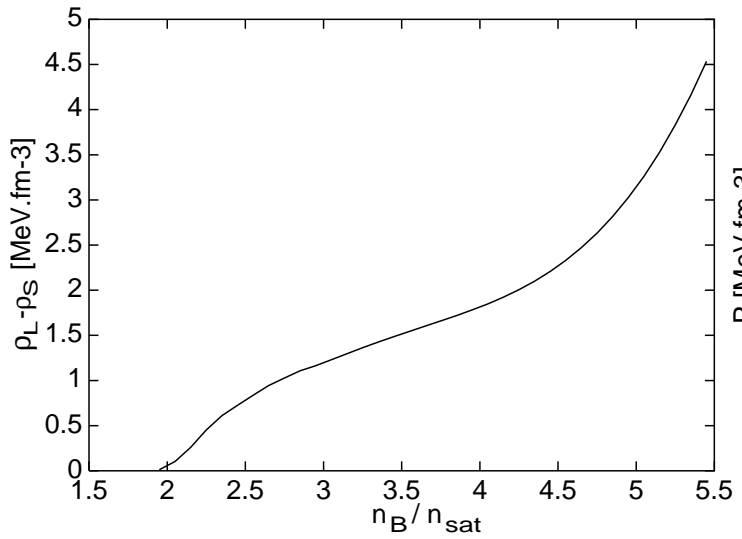

Figure 1. Energy difference between the liquid and crystallized phase, as a function of baryonic density

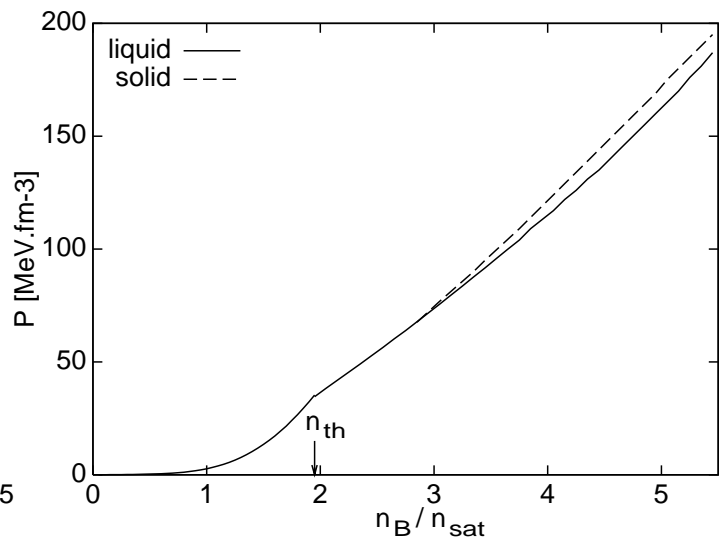

Figure 2. Pressure in the liquid and the crystallized phases, as a function of baryonic density.

$$
\begin{aligned}
\rho_{\Lambda} & =\left(M_{\Lambda}+\frac{3}{2} v_{0}+\Gamma_{\omega \Lambda}<\omega^{0}>+U_{0}\right) n_{\Lambda} \\
\rho_{\text {fields }} & =\frac{\Gamma_{\sigma N}}{2}\left(<\sigma>+<\sigma_{\Lambda}>_{s}\right)\left(n_{p}^{(s)}+n_{n}^{(s)}\right)+\frac{\Gamma_{\sigma \Lambda}}{2}<\sigma><n_{\Lambda}^{(s)}>_{s}+\frac{\Gamma_{\delta N}}{2}<\delta>\left(n_{p}^{(s)}-n_{n}^{(s)}\right) \\
& +\frac{\Gamma_{\omega N}}{2}\left(<\omega^{0}>+<\omega_{\Lambda}>_{s}\right)\left(n_{n}+n_{p}\right)+\frac{\Gamma_{\omega \Lambda}}{2}<\omega^{0}><n_{\Lambda}>_{s}+\frac{\Gamma_{\rho N}}{2}<\rho^{0}>\left(n_{p}-n_{n}\right)
\end{aligned}
$$

This energy is then compared to the energy density of the corresponding liquid phase $\rho_{L}$. The result is displayed in Figure 1. With the chosen model parameters we obtained an ordered phase which is energetically more favorable than the liquid one above the threshold for hyperon production $n_{\text {th }}=1.95 n_{\text {sat }}$. The shape of this curve is similar to the one which was obtained for parameter set $\mathrm{C}$ in [ [ 7 ], with however a somewhat smaller energy gain. The stability of the solid phase increases at high density.

Finally, we obtain the pressure from the thermodynamical relation

$$
P=n_{B}^{2} \frac{\partial\left(\rho / n_{B}\right)}{\partial n_{B}}
$$

The result is displayed in Figure 2. In contrast with the results obtained for parameter set $\mathrm{C}$ in [4], the equation of state of the ordered phase is now slighlty harder than the liquid one.

\section{CONCLUSION}

We investigated the possible formation of an ordered phase of the baryonic matter present in the core of the neutron stars, in which the hyperons are located on the nodes of a cubic lattice. The model presented in this short contribution is a first upgrade 
of our original barebones model [ 4 ]. We now have a more realistic description of the nuclear interaction, in particular of the nucleon sector. Our main conclusion is that the findings of [4] are confirmed, namely the ordered phase can be energetically more favorable for some choices of the model parameters. As could be expected, our result is found to depend more strongly on the features of the - largely unknown in-medium hyperon-hyperon potential than on the properties of the nucleon background such as incompressibility modulus and asymmetry. A more systematic study with several alternative parametrizations is underway and will be presented in a further publication.

The model which we have presented here can be improved in several ways. In order to be consistent with the DDRH picture, we described the hyperon-hyperon interaction by sigma and omega exchange with density dependent couplings in the form $\Gamma_{\alpha \Lambda}\left(n_{N}, n_{\Lambda}\right)=$ $x_{\alpha \Lambda} \Gamma_{\alpha N}\left(n_{N}+n_{\Lambda}\right)$ with $\alpha \in\{\sigma, \omega\}$. On the other hand, some authors [9] also introduce additional $\sigma *$ and $\phi$ exchange in order to reproduce the attractive $\Lambda-\Lambda$ interaction inferred from the available data on double hypernuclei. Furthermore, parametrizations of the free hyperon-nucleon and hyperon-hyperon potentials consider the exchange of e.g. kaons or $\eta$ which are also not present in our calculations.

Ideally one could extract an in-medium hyperon-hyperon potential from Bruecknertype calculations and test the result comparing with the available hypernuclei data. Whereas several non relativistic calculations exist in the litterature [10, 11], the corresponding calculations have not been performed in a relativistic framework. In particular a full fledged relativistic calculation including all hyperons (not only $\Lambda$ but also $\Sigma$ and $\Xi$ ) and a subsequent DDRH-type parametrization would be especially welcome. It would allow us to have a greater variety of lattice configurations involving in particular the $\Sigma^{-}$ hyperon. Indeed the $\Sigma^{-}$is formed in the liquid phase at the same density as the $\Lambda$ in most neutron star matter calculations.

An other direction of research would be to release the Sommerfeld approximation and take into account the redistribution of nucleons. This involves considering screening correlations of the RPA type to the potential.

Acknowledgment: This work was partially supported by project MCT-00-BFM0357.

\section{REFERENCES}

1. Haensel, P., “,” in Relativistic Gravitation and Gravitational Radiation, edited by J.-A. Marck and J.-P. Lasota, Proceedings of Les Houches School of Physics, Cambridge University Press, 1997.

2. Bonazzola, S., Private communication (2002).

3. Baiko, D.-A., and Haensel, P., Acta Phys. Polon. B, 30, 1097 (1999).

4. Pérez-Garcia, M.-A., Corte-Rodríguez, N., Mornas, L., Suárez-Curieses, J.-P., and Diaz-Alonso, J., Nucl. Phys. A, 699, 939 (2002).

5. Hofmann, F., Keil, C., and Lenske, H., nucl-th/0007050 (2000).

6. Keil, C., Hofmann, F., and Lenske, H., Phys. Rev. C, 61, 064309 (2000).

7. Hofmann, F., Keil, C., and Lenske, H., Phys. Rev. C, 64, 025804 (2001).

8. Typel, S., and Wolter, H., Nucl. Phys. A, 656, 331 (1999).

9. Schaffner, J., Dover, C., Gal, A., Millener, D., Greiner, C., and Stöcker, H., Ann. Phys. (N.Y.), 235, 35 (1994).

10. Baldo, M., Burgio, G.-F., and Schulze, H.-J., Phys. Rev., 61, 055801 (2000).

11. Vidaña, I., Polls, A., Ramos, A., Engvik, L., and Hjorth-Jensen, M., Phys. Rev., 62, 035801 (2000). 